\title{
Three-dimensional ultrasound imaging of the pressure ulcer. A case report.
}

\author{
Koichi Yabunaka1, Shinji Iizaka', Gojiro Nakagami², Masayuki Fujioka1 ${ }^{1}$, Hiromi Sanada ${ }^{1}$
}

${ }^{1}$ Department of Gerontological Nursing/Wound Care Management, Graduate School of Medicine, University of Tokyo, ${ }^{2}$ Department of Ultrasound, Katsuragi Hospital, Osaka, Japan

\begin{abstract}
We report the case of a 46-year-old female who presented with a category IV pressure ulcer (PU) in the sacral region. Undermining of the PU was assessed with the aid of two-dimensional and three-dimensional ultrasound (3D-US).3D-US clearly visualized the wound in three directions and allowed determination of its volume. Our results show that volumetric analysis carried out with 3D-US enables the evaluation of wound morphology and thus better treatment of patients with PUs. The technique is simple and can be used routinely in daily wound management to assess the volume of the undermined wound.

Keywords: three-dimensional ultrasound, two-dimensional ultrasound, pressure ulcer
\end{abstract}

\section{Introduction}

During the follow-up of patients with pressure ulcers (PUs), the wound size must be monitored precisely, together with the response to treatment [1-3]. However, it is difficult to accurately image the entire wound, especially when there is undermining, or tunneling, because the margins of an undermined region cannot be determined by visual assessment alone. A simple yet valid tool that allows PU outcomes to be measured in a meaningful way has yet to be developed.

Previous reports suggested the use of two-dimensional ultrasound (2D-US) to assess the pathogenesis of PUs [4-8], based on its ability to produce high-quality images of superficial tissue and its safety and utility in daily practice. However, 2D-US does not completely depict a PU in a single image. With three-dimensional ultrasound (3D-US) PUs can be imaged in their entirety such that the images can be used as a prognostic indicator [7] and

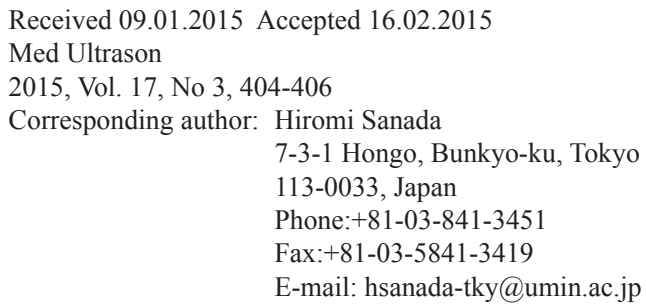

communicated to other, relevant caretakers (health professionals, parents).

The aim of this case report was to describe the use of 3D-US in a patient with undermining Pus, assessed with the aid of 2D-US and 3D-US.

\section{Case report}

A 46-year-old female presented with a category IV PU (undermining PU $1.5-2.5 \mathrm{~mm}$ in diameter) of the sacral region. Because the PU had persisted for more than 3 months, our hospital's PU team began management and follow-up for 5 weeks, at which time the patient left the hospital.

For ultrasonography evaluation the patient was placed in the lateral position. Sagittal 2D-US images were obtained in overlapping planes, and the resulting images were supplemented by transverse and oblique scans. Sonographic examinations lasted for approximately $5 \mathrm{~min}$. The PU surrounding the sacral region was scanned using a standardized scanning technique. The Aplio ultrasound system was used with a linear-array $(8.0 \mathrm{MHz}) 2 \mathrm{D}$ probe and a linear-array $\left(14 \mathrm{MHz}\right.$, swing angle $\left.60^{\circ}\right) 3 \mathrm{D}$ probe. The image depth during ultrasound imaging was $3 \mathrm{~cm}$. Echo gain and dynamic range were used to determine the proper display range. The probe was covered with ultrasound gel under a disposable wrap to avoid infec- 
tion. After 2D-US imaging the patient underwent 3D-US acquisition. 3D-US images were obtained in the sagittal plane using a 3D probe (Toshiba Medical Systems, Tokyo, Japan) and the same equipment as in 2D-US. The sweep scan, which automatically scans the area of interest, covered the target (the PU) and surrounding sacral region. The scan time was set for $5 \mathrm{~s}$. The obtained volume was stored on a hard disk and displayed later using dedicated software (4D View, Toshiba Medical Systems). Of the three planes available for image display [sagittal (A), axial (B), and coronal (C)], we selected plane A to $\mathrm{C}$ to trace the lesion margins. Multi-planar 3D-US images were reconstructed using volumes data to assess the wound size in a patient with an undermining PU.

The images obtained with 2D-US of the undermined PU revealed a marginal hypoechoic area with a smooth border and an internal spotty hyperechoic area with multiple high-echo spots, representing air, located within the subcutaneous fat layer at the sacral region (fig 1). The sagittal and axial planes clearly visualized the internal undermining and the shape of the lesion; however, in the coronal plane the entire PU could not be obtained in a

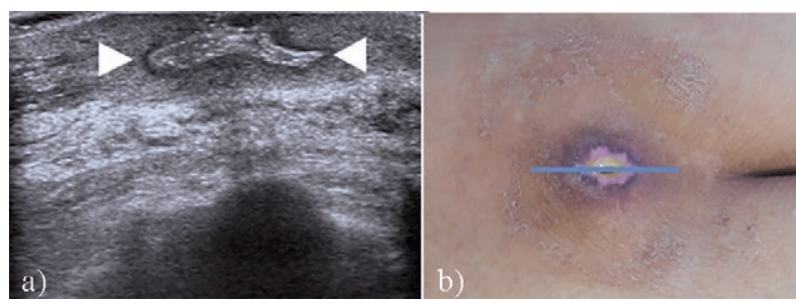

Fig 1. a) Longitudinal scan shows US imaging of the hyperechoic area with multiple high echo spot inside the undermining (arrowheads), b) the clinical aspect of the PU in the sacral region. *Blue line = observation point of ultrasonography

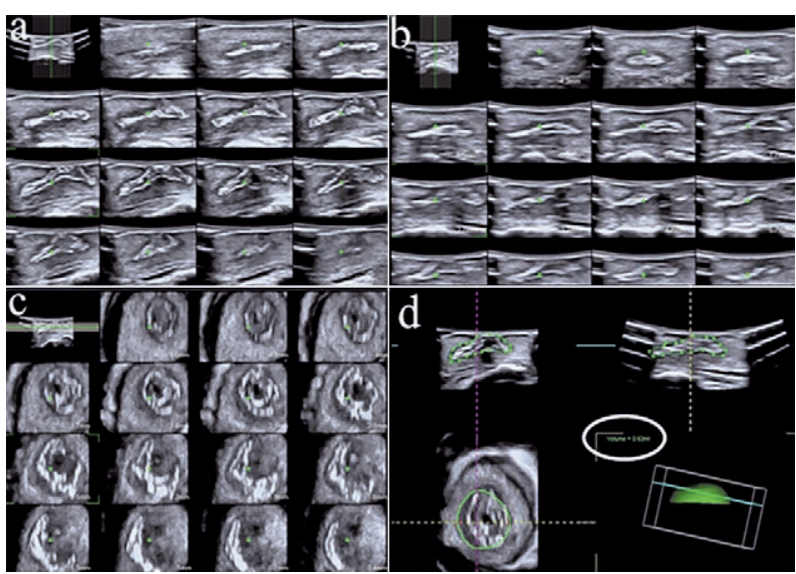

Fig 2. Multi-planar reconstructed 3D-US showing multi-view images for the whole undermined PU. The figure is visualized in three planes; sagittal (a), axial (b), and coronal (c); d) based on 3D-US images a volume of $0.63 \mathrm{ml}$ was calculated (oval). single 2D-US image (fig 2 a,b,c). 3D-US depicted the relationship between the undermining and the boundary of the normal skin tissue. Volume-rendered 3D-US images indicated a volume of $0.63 \mathrm{ml}$, based on the traced marginal region in the sagittal and axial planes (fig 2d).

The US examinations were performed by a certified sonographer (with more than 20 years of experience) experienced in the techniques required to assess skin and subcutaneous tissue. The study was approved by the Ethics Committee of our institution (\#031), and written informed consent was obtained from the patient.

\section{Discussions}

Our case study demonstrates the advantages of 3DUS as a novel method to image undermining PUs, as it provides important and unique information. The technique is simple and can be easily used in followup evaluations. As demonstrated in the present report, 3D-US yields clear volume-rendered three-dimensional images and allows determination of the volume of an undermined PU.

2D-US can be used to estimate tissue damage in localized sites of inflammation and will reveal whether a PU is cystic, solid, or mixed [5,7].Another application of 2D-US is in monitoring the tumor size, the extension into adjacent structures, and evidence of PU. However, 2D-US does not provide multiple continuous images in multi-directional planes. While both computed tomography (CT) and magnetic resonance imaging (MRI) can penetrate the entire depth of the region, they are relatively expensive to perform. Moreover, neither technique has been used to show a detailed body-surface area [9]. An additional disadvantage of $\mathrm{CT}$ is that it exposes patients to ionizing radiation, whereas MRI is contraindicated in patients with pacemakers, ferromagnetic implants, or severe claustrophobia.

Recently, several studies have examined the role of 3D-US in the evaluation of superficial tissue [10-12] and in the monitoring of changes in the tumor volume $[13,14]$. In this study, multi-planar reconstructed 3D-US images provided accurate images of the undermining's extension into adjacent structures and of its internal properties. Furthermore, with 3D-US both the volume of the undermined PU and the relationship of the ulcer to the subcutaneous tissue and muscle could be determined. This information may be of value to other care takers involved in the management of the patient. 3D-US is also useful in prenatal diagnosis and the resulting images provide important information when counselling parents. Health professionals will find 3D-US imaging technically simple to perform. 
There are two important limitations to the widespread adoption of 3D-US in patients with PUs: 1) large PUs cannot be effectively imaged and 2) there is no portable unit, which make it difficult to perform bed-side 3D-US.

In conclusion, 3D-US provides a volumetric analysis of PUs and allows evaluation of their morphology, thereby improving treatment. The technique is simple and can be used routinely in daily wound management to assess the volume of the undermined wound.

Acknowledgement: Volume-rendered 3D-US images obtained in this study were submitted to be considered for a prize for the best image of 2014, awarded by Toshiba Medical Systems Corporation Japan. We thank the Director of the Katsuragi Hospital, Dr. Mutsumi Ohuewho provided advice during the study period.

\section{References}

1. Phillips L, Clark M. Can meaningful quality benchmarks be derived from pressure ulcer prevalence data? J Tissue Viability 2010; 19: 28-32.

2. Harris C, Bates-Jensen B, Parslow N, Raizman R, Singh M, Ketchen R. Bates-Jensen wound assessment tool: pictorial guide validation project. J Wound Ostomy Continence Nurs 2010; 37: 253-259.

3. Beam JW. Topical silver for infected wounds. J Athl Train 2009; 44: 531-533.

4. Nagase T, Koshima I, Maekawa T, et al. Ultrasonographic evaluation of an unusual peri-anal induration: a possible case of deep tissue injury. J Wound Care 2007; 16: 365-367.
5. Aoi N, Yoshimura K, Kadono T, et al. Ultrasound assessment of deep tissue injury in pressure ulcers: possible prediction of pressure ulcer progression. Plast Reconstr Surg 2009; 124: 540-550.

6. Yabunaka K, Iizaka S, Nakagami G, et al. Can ultrasonographic evaluation of subcutaneous fat predict pressure ulceration? J Wound Care 2009; 18: 192-196.

7. Ueta M, Sugama J, Konya C, et al. Use of ultrasound in assessment of necrotic tissue in pressure ulcers with adjacent undermining. J Wound Care 2011; 20: 503-504, 506-508.

8. Higashino T, Nakagami G, Kadono T, et al. Combination of thermographic and ultrasonographic assessments for early detection of deep tissue injury. Int Wound J 2014; 11: 509516.

9. Gunnarsson U, Strigård K. 3D intrastomal ultrasonography, an instrument for detecting stoma-related fistula. Tech Coloproctol 2012; 16: 233-236.

10. Deng J, Newton NM, Hall-Craggs MA, et al. Novel technique for three-dimensional visualisation and quantification of deformable, moving soft-tissue body parts. Lancet 2000; 356: $127-131$.

11. Strigård K, Gurmu A, Näsvall P, Påhlman P, Gunnarsson U. Intrastomal 3D ultrasound; an inter- and intra-observer evaluation. Int J Colorectal Dis 2013; 28: 43-47.

12. Girtler MT, Krasinski A, Dejaco C, et al. Feasibility of 3D ultrasound to evaluate upper extremity nerves. Ultraschall Med 2013; 34: 382-387.

13. Presti F, Sanusi FA, Hamid R. Three-dimensional prenatal ultrasound study of a large sacrococcygeal teratoma. Int J Gynaecol Obstet 2001; 73: 61-63.

14. Bonilia-Musoles F, Machado LE, Raga F, Osborne NG, Bonilla F Jr. Prenatal diagnosis of sacrococcygeal teratomas by two- and three-dimensional ultrasound. Ultrasound Obstet Gynecol 2002; 19: 200-205. 